\title{
Lisa Yashodhara Haller und Alicia Schlender. Hg. (2022): Handbuch Feministische Perspektiven auf Elternschaft
}

\author{
Opladen: Barbara Budrich Verlag. 632 Seiten. ISBN: 3847423673. \\ Preis: 59,90 €
}

\section{Sarah Eckardt}

Angenommen: 27. Januar 2022

(C) Der/die Autor(en) 2022

Elternschaft, die Frage wie man schwanger wird und das Thema Geburt blieben lange Zeit eine ausgesparte Perspektive in der feministischen Auseinandersetzung. Der, in Europa bis in die Gegenwart hinein, tonangebende Gleichheitsfeminismus orientiert sich an der Gleichheit mit Männern - ungebunden und meist durch Frauen von Fürsorge befreit. Da Abhängigkeiten der Selbstbestimmung von Frauen Grenzen setzen, waren Debatten rund um die Geburt und Mutterschaft als privates Problem einiger Frauen - nämlich derjenigen, die Mütter wurden - marginal. Themen rund um Schwangerschaft, Geburt und Elternschaft blieben in den feministischen Debatten lange Zeit Gegenstand der Abgrenzung. Doch das scheint sich aktuell zu ändern. Ein Bedeutungswandel in der Perspektive auf Elternschaft hält Einzug in feministische Auseinandersetzungen und Kämpfe. Nicht länger richten sie sich gegen abhängige Lebenslagen, sondern gegen Verhältnisse, in denen das Leben mit Kindern zur Zumutung wird. Damit sind Schwangerschaft, Geburt, Feminismus und Familie in dieser Kombination ein neuer Untersuchungsgegenstand, dem in den letzten Jahren zunehmend wissenschaftliche Aufmerksamkeit geschenkt wird.

Das erste Handbuch im deutschsprachigen Raum zu feministischen Perspektiven auf Elternschaft, herausgegeben von Lisa Yashodhara Haller und Alicia Schlender, versammelt anhand von Schlagworten 50 Beiträge, die in fünf Rubriken untergliedert sind. In der ersten Rubrik geht es um die Fragen, wie Mutterschaft, Vaterschaft und Elternschaft in unterschiedlichen feministischen Theorieströmungen bearbeitet werden. Gesellschaftliche und staatliche Einflüsse auf das Leben mit Kindern, rechtliche Aspekte und die institutionelle Einbettung der Ausübung von Mutter- und Vaterschaft sowie deren Anwendung in unterschiedlichen Handlungsfeldern, u. a. der

Sarah Eckardt $(\square)$

Fachbereich Pflege und Gesundheit, Hochschule Fulda, University of Applied Sciences, Leipziger

Straße 123, 36037 Fulda, Deutschland

E-Mail: sarah.eckardt@pg.hs-fulda.de 
Kinder- und Jugendhilfe, werden in der zweiten Rubrik in den Blick genommen. In der dritten Rubrik sind die vielfältigen Wege zur Schwangerschaft Gegenstand der Analyse. Berücksichtigt werden dabei auch Fehl- und Totgeburten sowie die Varianten der Anwendung der Reproduktionsmedizin. Die vierte Rubrik thematisiert das Leben mit Kindern und die Frage, wie es praktisch organisiert werden kann. Abschließend widmen sich die Beiträge in der fünften Rubrik den feministischen Utopien eines guten Lebens mit Kindern und analysieren, welche Herausforderungen aus der Perspektive einer feministischen Familienpolitik bestehen.

Neben dem großen Thema der Elternschaft erfährt auch die Geburt als Thema feministischer Auseinandersetzungen eine Revitalisierung. Die Geburt ist gegenüber der Elternschaft nur eine sehr kurze Passage, die den Übergang von Frauen* und Männern* zu Eltern und den Beginn eines neuen Lebens markiert. Mit der Schwangerschaft, spätestens mit der Geburt, beginnt oft eine Retraditionalisierung von Beziehungsmodellen. Die Zuständigkeit für das heranwachsende Leben, aber auch dessen Umsorgung wird dabei nach wie vor häufig der Mutter zugeordnet und von dieser übernommen. Die Geburt wird von unterschiedlichen Seiten auch als ein Moment gedeutet, der für die weitere Entwicklung des Kindes, der Familie und die Gebärende von großer Bedeutung ist, und wird damit sehr stark mit Sinn aufgeladen. Da die Geburt lange Zeit als natürlicher oder allenfalls medizinischer Prozess galt, schien ihre Gestaltung ausgeschlossen und aus ihr ließ sich eine spezifische elterliche Arbeitsteilung ableiten. Doch auch die Geburt kann nicht jenseits von gesellschaftlichen Einflüssen betrachtet werden. Außerdem ist das Ereignis der Geburt immer auch mit Kinderwunsch, Wochenbett, Kinderernährung sowie Konstrukten von Weiblichkeit, Mutter- und Vaterschaft verbunden. Es gibt keine Natur jenseits der Kultur. In der sozialwissenschaftlichen Forschung erhält der Themenbereich erst seit den 2010er-Jahren eine stärkere Aufmerksamkeit. Dabei ist die Geburt ein besonders spannendes Ereignis, denn sie erscheint als eine ontologische Konstante oder Naturgewalt, obgleich in ihr Kultur und Natur eng verwoben sind. Das Erleben von Geburt kann für Gebärende (über-)mächtig sein, darum ist es hier besonders interessant, sie aus feministischer Perspektive zu betrachten und dabei den konzeptuellen und argumentativen Dualismus von Natur und Kultur, ein Gegeneinander von „natürlichen“ und medizinisch-technokratischen Geburtsmodellen neu zu akzentuieren. Auch das Handbuch versammelt einige Beiträge rund um das Thema Geburt. In Anlehnung an das Schwerpunktheft greife ich fünf besonders bedeutsame Beiträge heraus. Sie verhandeln Mutterschaft, Schwangerschaft, Geburt und Stillen zwischen Naturalisierung und Gestaltbarkeit, zeigen Forschungsstränge, theoretische Ansätze, Diskussionen und aktuelle Forschungsergebnisse.

Der Beitrag „Mutterschaft“ von Helga Krüger-Kirn eröffnet das Handbuch. Den Ausgangspunkt für eine kritisch-emanzipative Erforschung von Mutterschaft bildet eine historische Rekonstruktion von Mutterschaft und Mütterlichkeit als sozialhistorische Rollenbilder und kulturelle Imaginationen. Die Entwicklung der Reproduktionstechnologien hat zu weitreichenden Verschiebungen im diskursiven Feld von Schwangerschaft und Mutterschaft geführt. Krüger-Kirn zeichnet die Verbindung zwischen differenzfeministischen, gleichheitsfeministischen und poststrukturalistischen Ansätzen mit Mutterschaft nach. Abschließend werden zu jedem Ansatz die Herausforderungen und Probleme benannt, die aus den jeweiligen Perspektiven fol- 
gen. Krüger-Kirn plädiert dafür, das körperliche und psychische Erleben stärker $\mathrm{zu}$ fokussieren und damit auch vertieft den Zusammenhang von Reproduktion und weiblichem Körper herauszuarbeiten, beispielsweise die Schwangerschaft ,als zwischenleiblichen Prozess zu denken" (S. 20).

Die gesellschaftlichen, politischen und feministischen Herausforderungen, die sich aus der Tatsache ergeben, dass zwar alle Menschen geboren werden, aber nur etwa die Hälfte von ihnen schwanger wird, also selbst Menschen gebären kann, stellt sich Antje Schrupp in ihrem Beitrag „,Schwangerwerdenkönnen“. Die traditionellen (patriarchalen) Geschlechterordnungen haben diese Herausforderung dadurch gelöst, dass sie Menschen entlang dieser reproduktiven Differenz in unterschiedliche Geschlechterkategorien einteilten und entsprechende Rollenmuster und Verantwortlichkeiten ausprägten, die insgesamt zulasten derjenigen Menschen, die schwanger werde können - die „Frauen“ -, ging. Diese Praxis ist durch die Frauenbewegung infrage gestellt worden, womit sich allerdings die Notwendigkeit ergibt, andere, freiheitliche Umgangsweisen mit der reproduktiven Differenz zu finden, zum Beispiel im Hinblick auf Elternschaft jenseits von heteronormativen Paarbeziehungen, aber auch etwa bei ethischen Diskussionen über Reproduktionstechnologien. Dazu entfaltet der Text am Ende einige Vorschläge.

Obwohl stark institutionalisiert und fester Bestandteil der Geburtsvorbereitung, sind ,Geburtsvorbereitungskurse“ im deutschsprachigen Raum kaum erforscht. Marion Müller fokussiert sie in ihrem Beitrag in ihrem historischen Entstehungszusammenhang. Hebammengeleitete Geburtsvorbereitungskurse fungieren in Deutschland seit den 1990er-Jahren als Begleitinstitutionen des Übergangs zur Eltern- bzw. Mutterschaft. Im Rahmen wöchentlicher Treffen erhalten werdende Eltern Informationen über Schwangerschaftsverlauf, Geburt und die Zeit danach. Da Frauen die primären Adressatinnen sind und die Kurse vielfach in Form reiner Frauengruppen stattfinden, lassen sie sich als pränatale Instanzen zur Sozialisation und Vorbereitung auf die neuartigen Erwartungen als Mutter deuten. Damit könnten sie auch zu einer Vergeschlechtlichung von Elternschaft und der Übertragung von elterlicher Verantwortung auf die Schwangeren beitragen. Die Kurse sind nach Müller hauptsächlich auf das Wohl des Kindes und eine gelingende Mutter-Kind-Bindung im Verlauf der Geburt ausgerichtet, sie appellieren an die Leidensbereitschaft der werdenden Mutter. In Geburtsvorbereitungskursen greifen Informalisierung, Intimitisierung und Moralisierung ineinander. Durch sie und weitere Bestandteile der Geburtsvorbereitung (z.B. durch Ratgeber) werden Geburt und Mutterschaft idealisiert, gleichzeitig „werden die Frauen systematisch verunsichert“ (S. 344).

Tina Jung liefert einen umfangreichen und dichten Beitrag zum Thema „Geburt“. Zunächst zeichnet sie ,die gesellschaftliche Organisation von Geburt im historischen Wandel“" (S. 347) nach und beschreibt damit die zentralen Entwicklungslinien von Verwissenschaftlichung, Professionalisierung, Hospitalisierung, Medikalisierung, Humanisierung und schließlich Ökonomisierung der Geburt. Auch auf die gegenwärtige Situation, in der sich das Hebammenwesen durch das Hebammenreformgesetz 2020 akademisiert und Elterninitiativen sich für eine Verbesserung der Geburtshilfe einsetzten, geht Jung ein. Danach werden (sozial-)wissenschaftliche Perspektiven auf Geburt skizziert und ausgewählte Achsen feministischer Debatten dargelegt. Geborenwerden und Gebären verweisen darauf, dass Menschen ,,abhängig 
und beziehungsgebunden“ (S. 351) sind, und betonen die Bedeutung des Körpers. Auch die Bedeutung von Macht und Ohnmacht für Geburtskulturen und Gebären werden dargestellt und diskutiert. Besonders bei dem Thema der Geburt wird deutlich, dass die Aufgabe der sozialwissenschaftlichen Forschung darin besteht „die komplexen Verschränkungen von normierenden, ideologischen, politischen, praktischen, institutionellen und körperlichen Erfahrungen hinsichtlich der jeweils enthaltenen Dynamiken macht- und herrschaftskritisch zu untersuchen“ (S. 355f.). Jung kritisiert das Suggerieren der guten Planung als Garant für eine gute Geburt. Das Versprechen, durch individuell zu leistende Vorbereitung, gute Planung und richtig getroffene Entscheidungen könne der Geburtsvorgang optimal ausgestaltet werden, gehe an der Realität der gegenwärtigen Geburtshilfe vorbei (S. 354). Abschließend verweist Jung auf die globalen und intersektionalen Dimensionen des Gebärens, das sie bezugnehmend auf Miller auf die Kurzformel bringt ,,too much to soon“ für die Medikalisierung der Geburt im globalen Norden sowie ,too little to late " für die Unterversorgung während der Geburt im globalen Süden“ (S. 355).

Stillen gilt als beste Form der Säuglingsernährung. Der Beitrag von Lotte Rose und Eva Tolasch rekonstruiert die politische Historie der wechselvollen Etablierung des Stillens und liefert aktuelle Daten zur Stillbereitschaft. Im Anschluss wird auf Grundlage umfangreicher Forschung der Gender Studies zum Stillen der Frage nachgegangen, welche Folgen das Still-Ideal für die Vergeschlechtlichung der privaten elterlichen Fürsorgeverhältnisse hat. Dabei geht es um die Problematisierungen der mütterlichen Belastungen durch die Still-Norm sowie die väterliche Marginalisierung im Kontext des Stillens.

Das „Handbuch zu feministischen Perspektiven auf Elternschaft“ bietet insgesamt eine bereichernde Lektüre für Expert*innen, Einsteiger*innen oder Aktivist*innen. Es zeigt Einblicke in die breite Themenvielfalt rund um Elternschaft, die von vielen hochkarätigen Autor*innen diskutiert werden. Die Beiträge stellen den Wissensstand und aktuelle wissenschaftliche Debatten detailliert dar. Sie weisen pointiert auf zukünftige Herausforderungen für die Entwicklung neuer feministischer Konzepte, Denkmodelle und notwendiger empirischer Forschungsprojekte hin. Auch Geburt sowie Praktiken und Konzepte rund um Geburt werden betrachtet, was besonders gut gelingt. Durch das Handbuch können die unterschiedlichen Aspekte und Verstrickungen nach Belieben vertieft werden. Das umfangreiche Handbuch könnte jedoch noch ergänzt werden und sich stärker auf Geburt und Themen der reproduktiven Selbstbestimmung und Gewalt fokussieren. So schwingt beispielsweise das Gebären als aktive Leistung der werdenden Mütter in einigen Beiträgen mit, bleibt als eigener Gegenstand jedoch unerwähnt, ebenso wie das aktuelle und tagespolitisch viel besprochene Thema der Gewalt im Zuge der Geburtshilfe oder das Phänomen ,Regretting Motherhood“. Eine systematische Einbeziehung deutsch-deutscher Entwicklungen und die Auswirkung der Wiedervereinigung würden die Argumentationen bereichern. Auch eine vertiefte Betrachtung von der Herausbildung der Disziplin von Gynäkologie und Geburtshilfe sowie dem Hebammenwesen könnten das Buch ergänzen/vervollständigen. Trotz dieser Leerstellen bietet das Handbuch einen sehr gelungenen Überblick der feministischen Forschung zu Elternschaft und speziell zur Geburt, die stetig weiter an Bedeutung gewinnt. Die Lektüre lohnt sich in jeder Hinsicht. 
Funding Open Access funding enabled and organized by Projekt DEAL.

Open Access Dieser Artikel wird unter der Creative Commons Namensnennung 4.0 International Lizenz veröffentlicht, welche die Nutzung, Vervielfältigung, Bearbeitung, Verbreitung und Wiedergabe in jeglichem Medium und Format erlaubt, sofern Sie den/die ursprünglichen Autor(en) und die Quelle ordnungsgemäß nennen, einen Link zur Creative Commons Lizenz beifügen und angeben, ob Änderungen vorgenommen wurden.

Die in diesem Artikel enthaltenen Bilder und sonstiges Drittmaterial unterliegen ebenfalls der genannten Creative Commons Lizenz, sofern sich aus der Abbildungslegende nichts anderes ergibt. Sofern das betreffende Material nicht unter der genannten Creative Commons Lizenz steht und die betreffende Handlung nicht nach gesetzlichen Vorschriften erlaubt ist, ist für die oben aufgeführten Weiterverwendungen des Materials die Einwilligung des jeweiligen Rechteinhabers einzuholen.

Weitere Details zur Lizenz entnehmen Sie bitte der Lizenzinformation auf http://creativecommons.org/ licenses/by/4.0/deed.de.

Sarah Eckardt ist wissenschaftliche Mitarbeiterin im Forschungsprojekt „ELSA - Erfahrungen und Lebenslagen ungewollt Schwangerer" an der Hochschule Fulda. Die Soziologin promovierte innerhalb des Promotionskollegs „Familie im Wandel. Diskontinuität, Tradition und Strukturbildung“ an der FernUniversität Hagen zu Subjektivierungsweisen von gebärenden Frauen. Ihre Arbeitsschwerpunkte liegen im Bereich der Frauen- und Geschlechterforschung, Medizin- und Gesundheitssoziologie. 